Mots. Les langages du politique

$71 \mid 2003$

Mondialisation(S)

\title{
Des positions épistémologiques distinctes
}

\author{
Jacques Guilhaumou
}

\section{(2) OpenEdition}

Journals

Édition électronique

URL : https://journals.openedition.org/mots/8643

DOI : $10.4000 /$ mots. 8643

ISSN : 1960-6001

\section{Éditeur}

ENS Éditions

\section{Édition imprimée}

Date de publication : 1 mars 2003

Pagination : 172-176

ISBN : 2-84788-027-5

ISSN : 0243-6450

\section{Référence électronique}

Jacques Guilhaumou, "Des positions épistémologiques distinctes », Mots. Les langages du politique [En ligne], 71 | 2003, mis en ligne le 06 mai 2008, consulté le 23 avril 2022. URL : http:// journals.openedition.org/mots/8643; DOI : https://doi.org/10.4000/mots.8643

\section{(c) ENS Éditions}




\section{Des positions épistémologiques distinctes}

La publication récente de deux dictionnaires d'analyse du discours, de ses termes et de ses concepts nous renseigne sur le territoire occupé par un champ de recherche situé à la frontière de plusieurs disciplines, tout en étant fortement marqué par son ancrage dans la linguistique. Si les auteurs réunis à cette occasion se situent plus dans un réseau, avec ses multiples interconnexions, qu'au sein d'un mouvement unifié, il n'en reste pas moins que les ambitions affichées par les éditeurs méritent d'être prises au sérieux, d'autant plus qu'ils ont réalisé, avec l'aide des auteurs, un énorme travail, et d'une grande utilité pour l'ensemble de la communauté des chercheurs. Précisons d'emblée que nous sommes l'un de ses auteurs, au titre de la relation histoire-discours au sein du Dictionnaire d'analyse du discours.

Pour P. Charaudeau et surtout D. Maingueneau, la réalisation d'un Dictionnaire d'analyse du discours (désormais $D A D$ ) marque l'émergence d'une discipline qui aurait quelque peu mis de côté sa dimension critique initiale en s'élargissant «à l'ensemble des productions verbales», et qui aurait donc «développé un appareil conceptuel spécifique, fait dialoguer de plus en plus ses multiples courants et défini des méthodes distinctes » (entrée Analyse du discours, p. 45). Au dialogue ainsi entamé entre divers courants de l'analyse du discours, et qui fait la très grande richesse de cette publication, s'ajoute l'incitation auprès de chaque auteur à bien marquer, dans sa contribution, la perspective d'analyse du discours, par rapport aux points de vue de la linguistique, de la philosophie du langage, de l'analyse textuelle, etc., bref de l'ensemble des sciences humaines et sociales. Au-delà de ce dialogue fécond, un constat semblerait alors s'imposer: le passage de l'analyse du discours dans le camp des disciplines constituées marginalise sa dimension critique initiale, en tant que lieu d'interrogation et d'expérimentation. Pour notre part, nous avons soustitré notre ouvrage Discours et archive (Liège, Mardaga, 1994), écrit de concert avec D. Maldidier et R. Robin, Expérimentations en analyse du discours, ce qui revient à dire le contraire: la consolidation du champ de l'analyse de discours procède, selon nous, d'une interrogation historique et épistémologique permanente, ce qui suppose de revenir sans cesse sur le geste inaugural de l'analyse du discours, son inscription dans la matérialité de la langue.

En affirmant que l'analyse du discours n'est pas apparue, du moins en France, sur la base d'un acte fondateur, les éditeurs du $D A D$ contournent en partie cette réalité inaugurale, en laissant aux divers auteurs le soin d'y 
revenir ou non selon leur sensibilité au problème. Les choix épistémologiques des éditeurs de Termes et concepts pour l'analyse du discours (désormais $T C A D$ ), dictionnaire d'orientation praxématique donc inscrit dans la perspective de la production du sens, sont plus précis en la matière. Ils répondent mieux à notre attente épistémologique. L'entrée Épistémologie, absente du $D A D$, met ici l'accent sur la nécessité d'opérer un va-et-vient entre l'interrogation philosophique et l'expérimentation pratique, et dans le cas présent de s'interroger sur l'épistèmè des objets discursifs.

Ces auteurs n'hésitent donc pas, dans une entrée significativement intitulée Idéalisme et matérialisme en linguistique, d'une évidente résonance philosophique, à nous renvoyer aux fondements matérialistes de la langue, en soulignant alors que l'occultation de ce geste «réaliste» inaugural a des conséquences majeures dans les choix théoriques en analyse du discours. L'entrée Dialectique, présente dans les deux dictionnaires, souligne encore plus ce contraste: notion seulement argumentative et logique pour son auteur dans le $D A D$, elle constitue, pour celui de $T C A D$, une notion fondamentale pour comprendre l'articulation du langage au réel.

L'analyse de la production du sens à partir du repérage linguistique de ses marques dans le discours produit apparait ici singulièrement concernée par le primat ou non d'un point de vue matérialiste. Si quelqu'un parle, c'est que quelque chose existe: le langage est donc ontologiquement concerné par le réel, certes de manière dynamique, actionnelle. Une analyse du discours épistémologiquement fondée sur le statut matériel du langage nous semble, avec les auteurs de $T C A D$, et en dépit de leur champ restreint d'intervention, plus apte à répondre aux interrogations contemporaines sur les fonctionnements discursifs, que la confrontation des courants de l'analyse de discours avec des catégories discursives généralisantes définies au sein d'un espace disciplinaire.

Partant de la relation entre le langage et la praxis, terme là encore repris de la philosophie, mais reformulé dans un cadre linguistique, P. Siblot s'en prend à juste titre aux «théorisations régies pas un processus d'abstraction idéaliste» qui tendent à restreindre le champ de l'analyse du sens en refusant de prendre en compte la dimension linguistique des expériences pratiques d'appréhension et de transformation du monde dans sa matérialité. On peut se demander si la volonté de disciplinariser l'analyse de discours ne procède pas alors d'une certaine distanciation généralisante vis-à-vis des matériaux empiriques et de leurs ressources propres, par le fait d'une métacatégorisation qui tout à la fois dilue les notions de la linguistique et limite l'appréhension de l'historicité des textes. 
Un exemple parlant est celui de l'entrée Actualisation présente dans les deux dictionnaires. Pour D. Maingueneau, cette notion sert à désigner «la conversion, dans chaque prise de parole, du système linguistique en énoncé singulier». Et il ajoute significativement: "mais sa valeur reste instable». C'est en effet cette instabilité de la catégorie d'actualisation qui en fait, pour lui, une notion d'un intérêt limité pour l'analyste du discours. Après avoir situé cette notion du côté de la praxématique, il précise qu'elle a «l'inconvénient de se trouver au cœur des sujets les plus controversés de la réflexion contemporaine sur le langage»! À contrario, les auteurs de $T C A D$ donnent toute son amplitude à la notion d'actualisation définie comme ce qui permet de " passer des potentialités de la langue à la réalité du discours », inscrivant ainsi l'analyse du discours dans une perspective dynamique qui pose en permanence la question de la continuité/discontinuité entre la langue et le discours.

Il faut cependant reconnaitre que le clivage entre une vision statique et une approche dynamique des catégories de l'analyse du discours traverse les deux dictionnaires. Ainsi, de l'exemple de Contexte, défini, du côté de la praxématique, comme le simple fait de «la relation entre deux ordres de phénomènes qui s'informent mutuellement» alors que l'auteur du $D A D$ met l'accent sur la réflexion récente en la matière, qui tend à souligner le rôle processuel du contexte. Il en est de même de la notion de Corpus, fort importante pour pouvoir appréhender des phénomènes discursifs sur une vaste surface textuelle. Prise d'une part comme un simple geste technique de l'analyste du discours au moment du recueil des énoncés attestés, elle peut aussi d'autre part, pour l'auteure du $D A D$, se problématiser de telle façon qu'elle mette en jeu la conception même de la discursivité. C'est le cas tout particulièrement des corpus d'archive qui relèvent d'un geste de lecture ouvrant des perspectives nouvelles sur la dynamique des genres et les prises de parole des acteurs ordinaires, au-delà de l'accent mis au départ sur les discours fortement légitimés.

Il n'en reste pas moins que les catégories revendiquées comme pivots de l'analyse de discours dans le $D A D$ sont souvent les catégories les plus stables et les moins concernées par les débats entre linguistes. Nous serions alors confrontés à une discipline pacifiée, donc stable. Ce choix peut s'avérer dans certains cas très problématique, tant dans le rapport à la tradition linguistique, que dans la relation aux débats actuels. Ainsi pourquoi s'en tenir d'un côté à la seule notion de Champ discursif, catégorie ad hoc de l'analyse de discours, alors que, de l'autre côté, il est question de façon certes plus classique de Champ, Champ lexical, Champ sémantique, Champ notionnel, notions dont l'analyste de discours a usé de façon cou- 
rante dans ses descriptions textuelles? De même, nous pouvons remarquer que l'entrée Définition, dans le $D A D$, ne fait pas référence au colloque de 1988 , tenu à l'Université Paris-Nord qui s'inscrit dans une perspective linguistique. Mais là aussi, du fait de la diversité des auteurs, notre remarque critique n'induit aucune systématicité. Par exemple, l'entrée Référence s'interroge d'emblée sur la place qu'occupe cette notion dans le débat philosophique et sémantique, l'interpellant donc à la fois dans son référent de réalité et sa manière de désigner une propriété du signe linguistique. Dans l'ensemble, le lien avec les débats actuels en lexicologie et en sémantique au sein de la linguistique est bien traité, ce qui s'avère beaucoup moins vrai pour les autres domaines des sciences du langage.

Il est un fait que la position de D. Maingueneau n'est pas celle de tous les auteurs du $D A D$ tant ils demeurent soucieux, pour une partie d'entre eux, de conserver le lien de la discursivité à la matérialité de la langue en tant que «lieu matériel ou se réalise des effets de sens», pour reprendre une expression de M. Pêcheux. La richesse de ce $D A D$ procède donc, audelà d'une catégorisation recherchant des valeurs stables en analyse du discours, de la grande diversité des champs abordés.

$\mathrm{Ne}$ faut-il pas alors considérer, pour reprendre une formule de S. Auroux, que «la sphère du concept c'est la sphère du sens »? Tout objet de l'analyse du discours ne relève donc pas nécessairement d'un positionnement au sens où $\mathrm{D}$. Maingueneau renvoie les catégories de champ, univers, espace discursif à une identité énonciative qui se constitue en son sein de façon stable. Cette approche très peu dynamique (pour ne pas dire très peu historique) de l'analyse du discours prend le risque de faire l'économie du rapport de l'énoncé à ses objets, ses sujets et ses concepts: cet espace corrélatif, selon M. Foucault (voir l'entrée Analyse archéologique), qui met en évidence des positions intrinsèques très variables au sein même de l'énoncé, et rend donc compte de «la délocalisation tendantielle du sujet énonciatif» pour reprendre une expression de M. Pêcheux. Tout se passe présentement comme si le discours généralisant de ce $D A D$ devait rendre disponible un certain nombre de catégories discursives dans le but de répondre au besoin de tel ou tel terme chez l'analyste de discours décrivant des pratiques discursives. Ne convient-il pas plutôt de s'intéresser aussi, en analyse de discours, aux conditions langagières de production des concepts qui permettent d'appréhender l'ensemble d'une réalité sociale par la médiation de la langue comme fait matériel, contexte et ressource?

Sous son apparence plus restreinte, le dictionnaire des Termes et concepts pour l'analyse du discours nous permet de mieux appréhender, 
certes d'un point de vue praxématique, les relations qui s'établissent entre les concepts au sein d'une analyse de discours qui demeurent bien sûr proche des objets et des sujets. Il fait ainsi contraste avec le Dictionnaire d'analyse du discours qui tend parfois à instrumentaliser le métadiscours des analystes du discours, pour une part auteurs de ce dictionnaire, et sans doute à leur corps défendant.

Jacques Guilhaumou

\section{L'entrée Doxa: pour un traitement rigoureux d'une notion floue}

La doxa est un bon exemple de ce que l'on peut appeler une notion floue, à la fois dans son contenu sémantique et ses usages en discours. Absente du TCAD, l'entrée Doxa figure dans le $D A D$, mais la définition qui en est donnée semble affectée de ce flou dans l'approche définitoire elle-même. Il est cependant possible, et même souhaitable, de traiter rigoureusement les notions floues, d'autant plus que des travaux récents et même plus anciens permettent une approche précise de la doxa, via une mise en relation nécessaire avec la notion de sens commun.

L'article du $D A D$, après une rapide définition de doxa, change d'objet, glissant vers les endoxa: l'auteur donne la définition d'Aristote dans les Topiques, signale le lien avec la notion d'autorité, et fait ensuite un commentaire sur la valeur péjorative affectée aux endoxa. Ce glissement implique une restriction du champ d'analyse et de fonctionnement de la notion: l'auteur la cantonne dans le domaine de l'argumentation (dialectique ou rhétorique). Par ailleurs, c'est surtout le problème de la péjoration affectant les endoxa qui est mentionné, et non leur rôle dans le fonctionnement des discours.

Dans la brève définition initiale, l'auteur définit la doxa comme «l'opinion, la réputation, ce que l'on dit des choses et des gens». Il signale que «la doxa correspond au sens commun», défini comme «un ensemble de représentations socialement prédominantes». Outre que l'emploi de correspondre ici peut laisser entendre une assimilation des deux notions, que les développements ultérieurs de l'article ne permettent pas de corriger, ces définitions passent sous silence l'importance à la fois historique, théorique et généalogique des deux notions.

Doxa, mot grec, est posé comme notion par Platon, en particulier dans La République. Il existe une excellente archéologie du terme et de la 